\title{
Results of Short-Term and Long-Term Pulmonary Vein Isolation for Paroxysmal Atrial Fibrillation Using Duty-Cycled Bipolar and Unipolar Radiofrequency Energy
}

\author{
MARCUS WIECZOREK, M.D., ${ }^{*}$ REINHARD HOELTGEN, ${ }^{*}$ ELVAN AKIN, M.D., ${ }^{*}$ \\ ALI REZA SALILI, M.D., ${ }^{*}$ HAKAN ORAL, M.D., F.A.C.C., $\dagger$ \\ and FRED MORADY, M.D., F.A.C.C. $\dagger$
}

From the *Department of Electrophysiology, Herzzentrum Duisburg, Germany; and $\dagger$ University of Michigan, Ann Arbor, Michigan, USA

\begin{abstract}
PV Isolation Using Bipolar/Unipolar RF Energy. Background: Electrical disconnection of the pulmonary veins (PV) plays an important role in the ablation of paroxysmal atrial fibrillation (AF). Antral ablation using a conventional steerable ablation catheter often is technically challenging and time consuming.

Methods: Eighty-eight patients (mean age $58 \pm 11$ years) with symptomatic paroxysmal AF underwent ablation with a circular mapping/ablation decapolar catheter (PVAC). Ablation was performed in the antral region of the PVs with a power-modulated bipolar/unipolar radiofrequency (RF) generator using 8-10 W delivered simultaneously through 2-10 electrodes, as selected by the operator. Seven-day Holter monitor recordings were performed off antiarrhythmic drugs at 3-, 6-, and 12-month follow-up, and patients were requested to visit the hospital in the event of ongoing palpitations. All follow-up patients were divided into 2 groups: Group 1 with a follow-up of less than 1 year and group 2 patients completing a 1-year follow-up.

Results: Overall, 338 of 339 targeted PVs (99\%) were isolated with the PVAC with a mean of $24 \pm 9$ RF applications per patient, a mean total procedure time of $125 \pm 28$ minutes, and a mean fluoroscopy time of $21 \pm 13$ minutes. Freedom from AF off antiarrhythmic drugs was found in 82 and $79 \%$ of group 1 and group 2 patients, respectively. No procedure-related complications were observed.

Conclusion: PV isolation by duty-cycled unipolar/bipolar RF ablation can be effectively and safely performed with a circular, decapolar catheter. Twelve-month follow-up data compare favorably with early postablation results, indicating stable effects over time. (J Cardiovasc Electrophysiol, Vol. 21, pp. 399-405, April 2010)
\end{abstract}

atrial fibrillation, catheter ablation, pulmonary vein isolation, unipolar/bipolar ablation, duty-cycled ablation

\section{Introduction}

Conventionally, pulmonary vein (PV) isolation for patients with atrial fibrillation (AF) is performed in a pointby-point manner using a single-tip catheter delivering unipolar radiofrequency $(\mathrm{RF})$ energy. This technique requires a high degree of operator skill and as a result the procedures can be lengthy and may be associated with extensive radiation exposure and procedural risks. In addition, creating reliable contiguous transmural lesions can be difficult with this approach.

This study describes the acute electrophysiological effects and follow-up results with a recently introduced technique for PV isolation by antral ablation using an over-the-wire, multielectrode catheter system that delivers duty-cycled bipolar

Drs. Morady and Oral report stock options, advisory board participation, and patent ownership in relation to Ablation Frontiers. Dr. Wieczorek is a consultant on training for CMC meetings for Ablation Frontiers and Medtronic. No other authors reported conflicts.

Address for correspondence: Marcus Wieczorek, M.D., Department of Electrophysiology, Herzzentrum Duisburg, Gerrickstrasse 21, D-47137 Duisburg. Fax: 00492034513209; E-mail: drmwieczorek@t-online.de

Manuscript received 28 June 2009; Revised manuscript received 18 August 2009; Accepted for publication 11 September 2009.

doi: 10.1111/j.1540-8167.2009.01640.x and unipolar RF energy simultaneously through up to 10 electrodes at relatively low power. ${ }^{1-5}$

\section{Methods}

\section{Patients and Procedure}

The study included 88 consecutive patients with drug refractory paroxysmal AF undergoing catheter ablation in our institution. All patients had symptomatic AF refractory to at least 2 antiarrhythmic drugs and underwent catheter ablation of AF after informed consent. They were screened with transthoracic and transesophageal echocardiography before ablation to rule out a significant reduction in left/right ventricular systolic function, left atrial enlargement greater than $50 \mathrm{~mm}$, or left atrial appendage thrombus.

All procedures were performed in the drug-free state, with the exception of amiodarone, and with fentany/midazolam to maintain conscious sedation. We used a recording system (EP-WorkMate, St. Jude Medical, St. Paul, MN, USA/EP Med Systems, West Berlin, NJ, USA) with filter settings of $100-500 \mathrm{~Hz}$ and maximum signal amplification for the PV ablation catheter (PVAC) (AblationFrontiers, Inc., Carlsbad, CA, USA). All other intracardiac bipolar signals were filtered between 30 and $500 \mathrm{~Hz}$ with variable signal amplification. No additional nonfluoroscopic mapping or navigation system was used. 


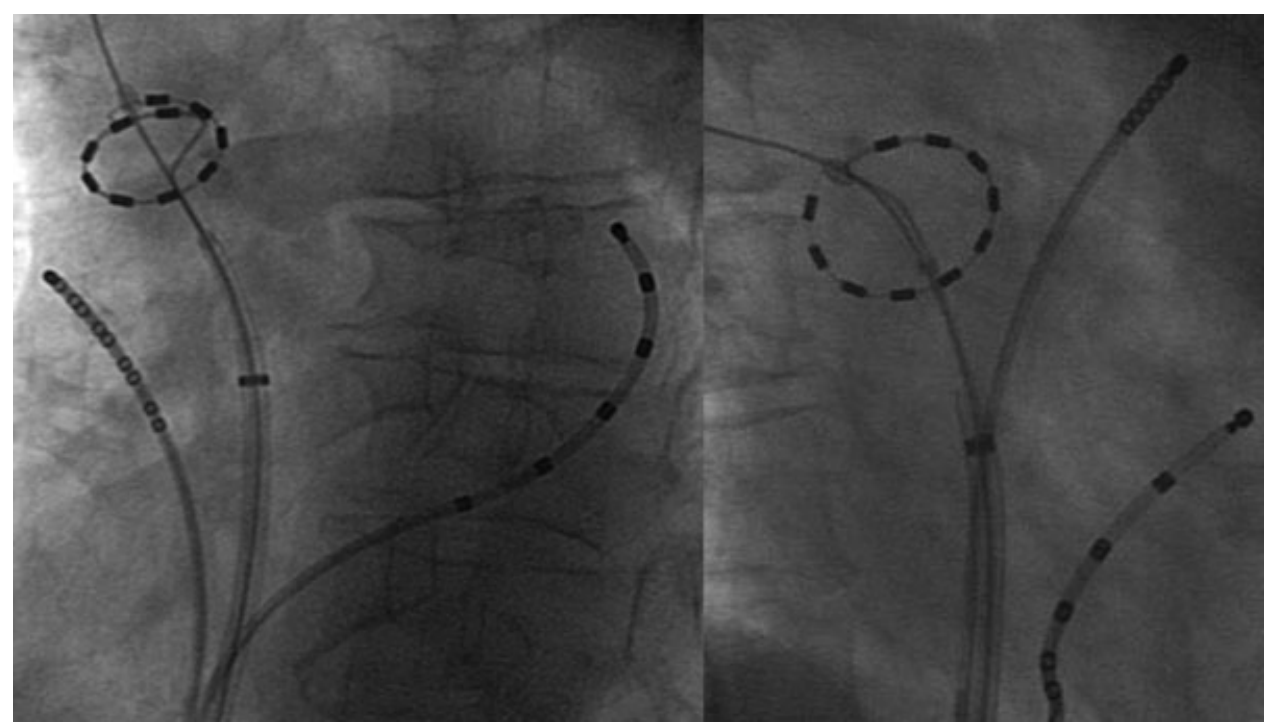

Figure 1. PVAC mapping of a right superior PV in LAO (a) and RAO (b) projections. (a) The PVAC was decreased in its diameter to map PV potentials within the vein. As a result, there is a visible overlap of the PVAC electrodes 1 and 2 with the electrodes 9 and 10. Clockwise rotation of the catheters decreases the PVAC diameter. (b) Counterclockwise rotation of the PVAC increases the diameter of the catheter in the antral region of this vein where RF ablation is performed. Note that electrodes 1 and 10 are now far apart. Further rotation of the PVAC after ablation is mandatory to create a continuous lesion by overlapping the lesions.

A hexapolar electrode catheter was placed in the coronary sinus (CS) for pacing and bipolar recordings. After transseptal puncture, a fixed-curve long $10 \mathrm{Fr}$ sheath (Frontier Advance; AblationFrontiers, Inc.) was inserted into the left atrium, and heparin was administered intravenously to maintain an activated clotting time of 300-350 seconds. Angiography was performed manually via the sheath to delineate all PVs. The PVAC was then advanced through the sheath into the left atrium to perform mapping and ablation of the targeted PVs. Later in the procedure, another multipolar steerable $6 \mathrm{~F}$ catheter was placed into the left atrium using the same access and used for differential pacing from several positions (left atrial appendage, posterior left atrial wall). This catheter was also placed into the right atrium to pace from different sites, if differentiation between right-sided PV potentials and far-field atrial signals was necessary. At the end of the procedure and after withdrawal of all catheters, an echocardiogram was performed in the EP laboratory to rule out pericardial effusion.

\section{Pulmonary Vein Ablation Catheter}

The PVAC is a 9 Fr, over-the-wire, circular, decapolar mapping and ablation catheter with a $25-\mathrm{mm}$-diameter array at the distal portion. ${ }^{1}$ The handle allows bidirectional deflection of the shaft and extension of the distal tip to form a spiral configuration. The platinum electrodes $(3 \mathrm{~mm}$ long, $1.5 \mathrm{~mm}$ outer diameter, $3 \mathrm{~mm}$ spacing) have a thermocouple under the surface on the anterior side. Mapping of the PVs is performed with 5 bipolar recordings through adjacent electrode pairs. The diameter of the electrode array can be effectively altered by engaging the shaft against anatomic structures and rotating the catheter shaft. Thus, clockwise rotation decreases the diameter to allow more distal positions inside the PV, whereas counterclockwise rotation increases the diameter of the array, facilitating antral ablation around larger veins (Fig. 1). The PVAC is positioned in the antral region of the targeted PV over a $0.032^{\prime \prime}$ guide wire, which is selectively placed into different side branches of the $\mathrm{PV}$ to improve the tissue-electrode interface around the PV circumference.

\section{GENius Multichannel RF Generator}

The GENius multichannel RF generator (Frontier Advance, AblationFrontiers, Inc.) was used for all ablation procedures. It is capable of independently delivering duty-cycled RF energy to a maximum of 12 electrodes simultaneously. $\mathrm{AC}$ power can be delivered in various combinations of unipolar and/or bipolar current. The unipolar energy consists of current flowing from the catheter electrode to the dispersive electrodes on the patient's back, and the bipolar energy consists of current flow between adjacent pairs of electrodes of the catheter array. Because electrodes 1 and 10 are far apart, the current flow between them is negligible. The generator offers 5 different preset energy settings: unipolar, bipolar, and different ratios of bipolar/unipolar energy in a 4:1, 2:1, and 1:1 fashion, respectively. Electrode pairs can be activated or deactivated for RF ablation selectively, depending on electrogram interpretation and tissue contact. During RF applications, energy delivery to individual electrodes is temperature controlled by a software algorithm that modulates power to reach the user-defined target temperature. It limits the power to a maximum of $8 \mathrm{~W}$ per electrode in the $4: 1$ ablation-mode and to a $10 \mathrm{~W}$ maximum in all other settings. Thus lesion depth and overlap between electrodes are controlled by varying the energy settings on the generator, with depth achievable up to $7 \mathrm{~mm}$ in the unipolar mode..$^{5,6}$

\section{Mapping and Ablation Protocol}

The PVAC was deployed in the left atrium over a $0.032^{\prime \prime}$ guide wire inside the PV and then advanced until it was in close contact with the antral region proximal to the ostium.

Contact was assessed by electrogram interpretation, operator sensation, and temperature during ablation. Stimulation was performed from different left atrial regions (distal CS 


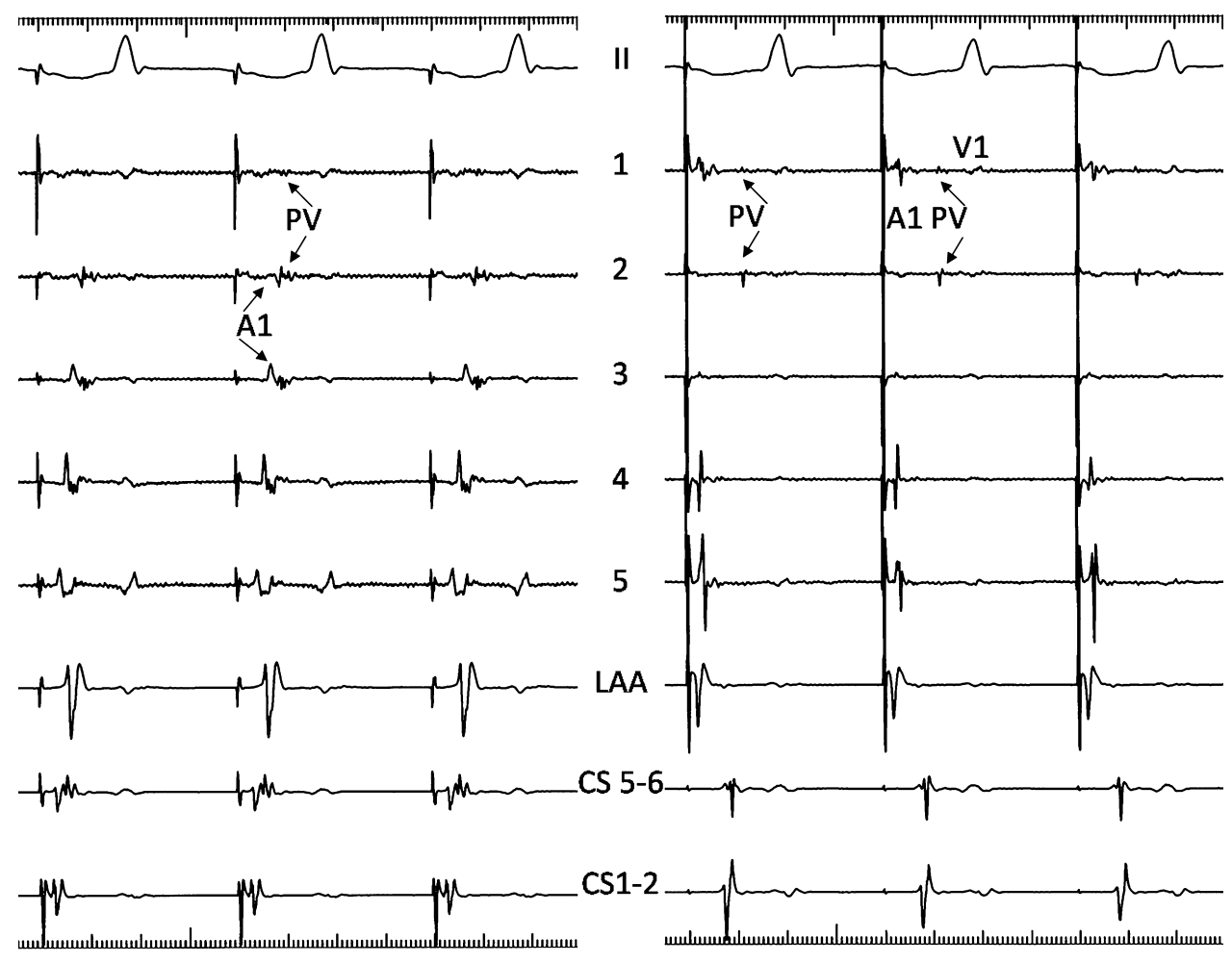

Figure 2. Intracardiac signals from PVAC in the antral region of a LSPV. Differential pacing from the distal coronary sinus (left) and LAA (right) to distinguish delayed antral conduction as a result of ablation from residual PV potentials. Detection of late and fragmented local electrograms from PVAC pair 1 and 2, when pacing is performed from the distal CS. Pacing from LAA clearly identifies these late signals in pair 1 and 2 as residual delayed PV potentials, whereas the split signal in pair 3 is now captured and advanced by pacing.

and left atrial appendage, posterior left atrium) and right atrial regions (right atrial appendage, posterior right atrial wall), for the detection of PV-potentials originating from the left- and right-sided PVs, respectively (Fig. 2). If no PV potentials were detected with the PVAC in the antral region, the catheter was further advanced into the PV ostium or inside the vein and the stimulation maneuvers were repeated. All veins showing PV-potentials spontaneously or as a result of pacing were targeted for antral ablation to achieve PV isolation. An optimal position for ablation was based on electrograms, with the goal of ablating local antral potentials of high amplitude on as many electrodes as possible for each application until all local potentials were abolished. Care was taken to avoid energy application inside the PVs. Common ostia were isolated by inserting the guide wire into the different side branches and ablating subsequent segments of the targeted veins.

Ablation in the antral region was always initiated in a 4:1 bipolar/unipolar ratio with all electrodes showing local electrical activity being activated to achieve contiguous transmural antral lesions, as far as possible, with a single catheter position. Later in the ablation procedure, RF applications in a 2:1 or even 1:1 mode were applied if PV isolation could not be obtained with the $4: 1$ setting. These applications were limited to individual electrodes showing local electrical activity to minimize collateral injury. Each energy application lasted 60 seconds and temperature was limited to $60^{\circ} \mathrm{C}$ for each electrode in the selected pairs. This approach was repeated for all targeted PVs until electrical disconnection of all veins was demonstrated. For mapping, the PVAC was repeatedly advanced into the PV ostium for mapping and stimulation as suggested by Iwasa et al. ${ }^{7}$ or inside the vein to show the elimination of all $\mathrm{PV}$ potentials or their electrical dissociation from the atrial activity (Fig. 3). PV isolation was defined as entrance block in all patients. If there were residual potentials near the PV ostium or inside the vein, differential pacing from different regions of the left and right atrium was performed to differentiate residual PV conduction from atrial far-field signals. In case of confirmed PV potentials, additional selective $\mathrm{RF}$ applications in the antral region of this vein were performed until isolation was complete (Fig. 4). If AF persisted during the ablation procedure and made the interpretation of the local electrograms obtained from the PVAC difficult, sinus rhythm was restored by external electrical cardioversion.

\section{Postablation Management}

At the completion of the procedure, anticoagulation with low molecular weight heparin was applied 2 hours after hemostasis was achieved and continued until the target INR (2.5) was reached when warfarin was administered. Anticoagulation with warfarin was continued in all patients for 3 months, and the patients were discharged from the hospital 2 days after the procedure. The last 12 patients treated with the PVAC underwent endoscopy of the esophagus by a gastroenterologist 24 hours after the ablation procedure.

\section{Follow-Up}

Patients were seen 3,6 , and 12 months after the ablation procedure in an outpatient clinic. Antiarrhythmic drug therapy was used in patients with early and recurrent episodes 


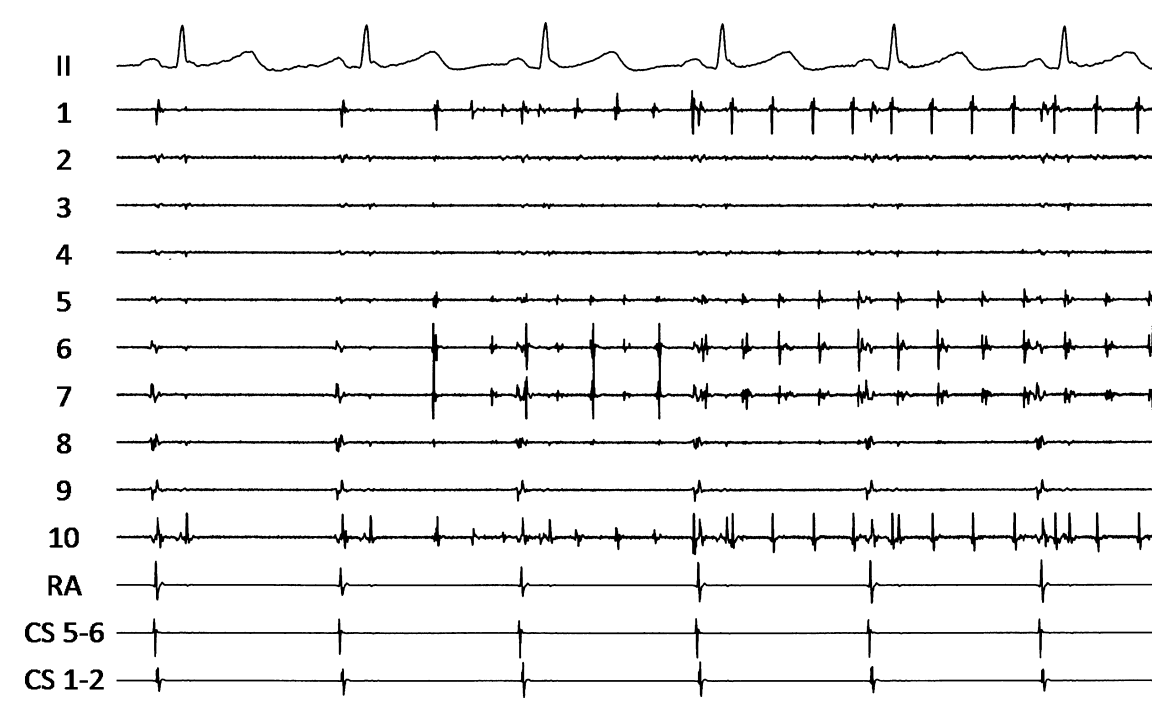

Figure 3. Bipolar electrograms recorded from the PVAC (1-10) placed within a left superior PV. Shown is the onset of a very rapid electrical activity of $160 \mathrm{~ms}$ within this vein with no conduction to the atria, since lead II and intracardiac recordings in the coronary sinus (CS) and right atrium (RA) demonstrate a dissociated regular slow atrial rhythm. This was recorded after antral ablation with the PVAC.

of symptomatic AF and discontinued after a blanking period of 3 months at least 3 days before the first follow-up evaluation. Therapy with amiodarone was discontinued at the time of ablation. Patients were requested to visit the hospital in the event of ongoing palpitations. Seven-day Holter monitor recordings were performed off antiarrhythmic drug therapy at each follow-up interval, starting 3 months after the initial procedure. Freedom from AF was defined as the absence of AF /flutter/tachycardia lasting $>30$ seconds after a single ablation procedure, in the absence of antiarrhythmic drug therapy.

Group 1 consists of all patients with a follow-up period of less than 1 year, while group 2 includes patients completing a 12-month follow-up. All patients who required a redo procedure within the follow-up period were classified as a primary failure of the initial ablation procedure.

At 6-month follow-up MRI was performed in 18 patients because AF ablation near the PVs is known to be a risk factor for PV stenosis, which was defined as PV diameter reduction of $30 \%$ or more.

\section{Results}

Table 1 shows the characteristics of the study group. The mean left ventricular ejection fraction was $61 \pm 6 \%$, and the mean left atrial diameter was $44 \pm 4 \mathrm{~mm}$. The percentage of patients with lone AF was $66 \%$.
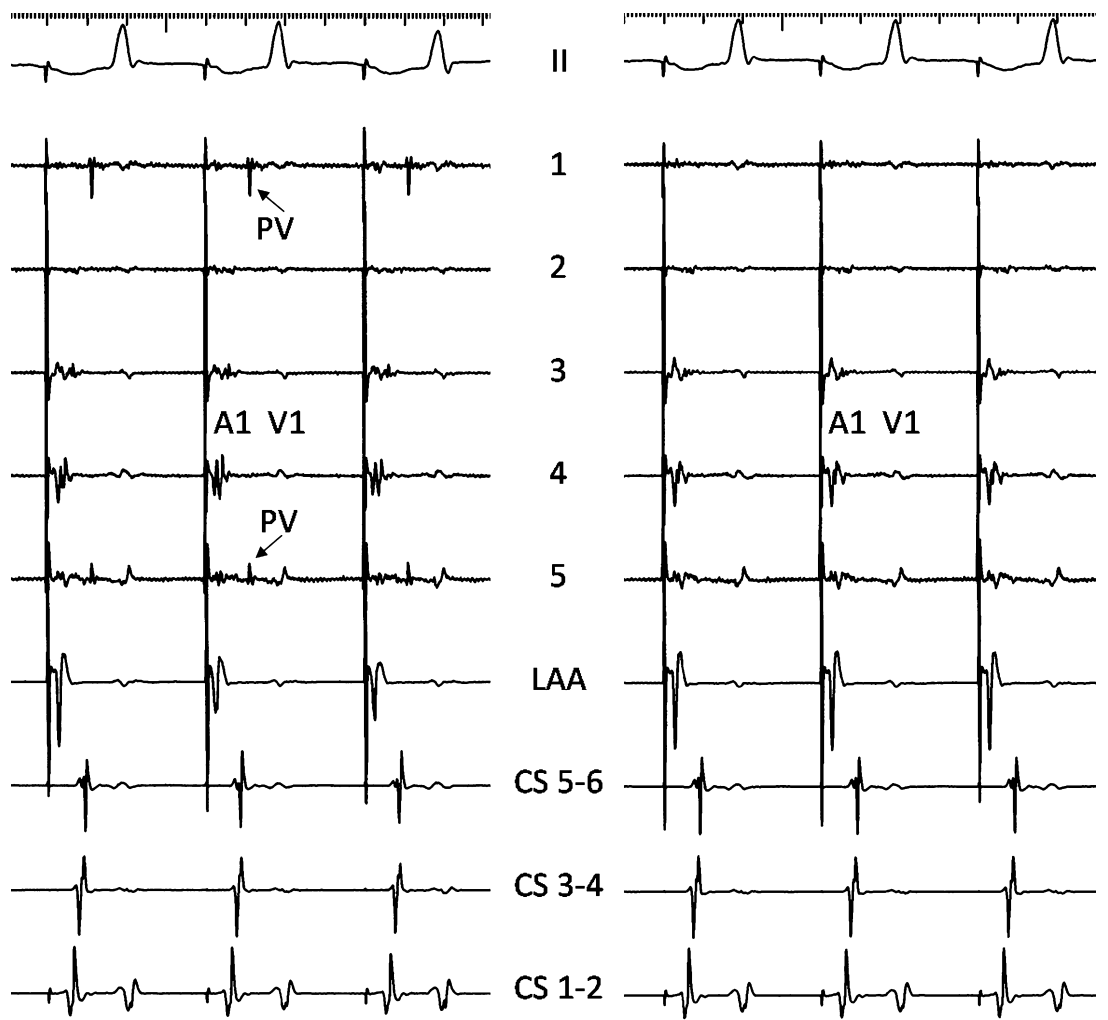

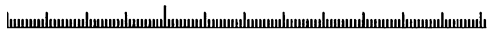

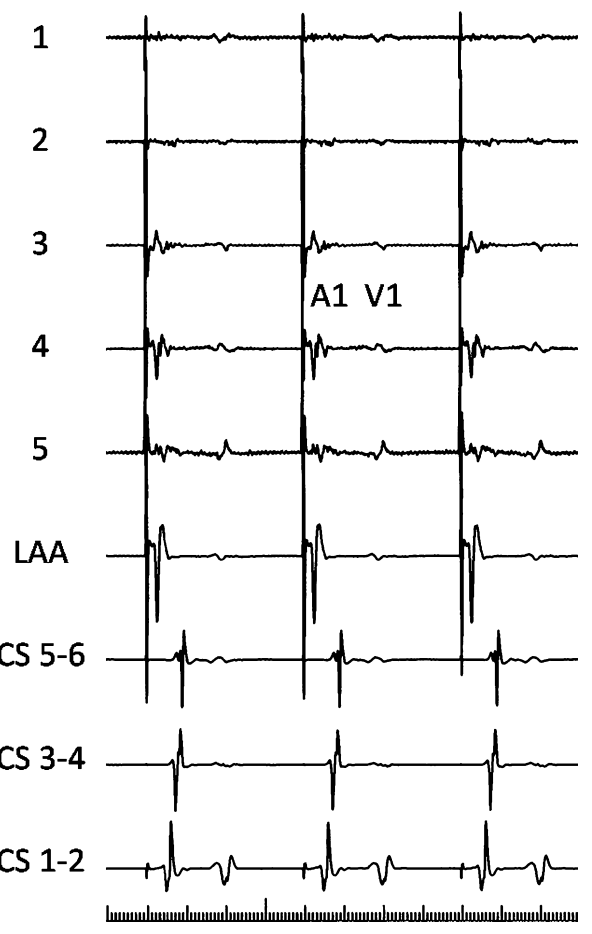

Figure 4. Antral PVAC mapping with 5 bipoles (1-5) of a left inferior PV before (left) and after ablation (right). Stimulation is performed from the LAA demonstrating fragmentation of the antral electrograms in all pairs as a result of previous energy applications. PV potentials are detected at pairs 1 and 5. Energy delivery for 60 seconds via the corresponding PVAC bipoles 1 and 5 with a 2:1 bipolar/unipolar ratio resulted in elimination of the PV potentials. 
TABLE 1

Patient Characteristics

\begin{tabular}{lc}
\hline No. of Patients & $\mathbf{8 8}$ \\
\hline Age (years) & $58 \pm 11(30-73)$ \\
Male/female & $55 / 33$ \\
Left atrial diameter (mm) & $44 \pm 4(35-49)$ \\
No. of antiarrhythmic drugs & $1.8 \pm 1.3(1-4)$ \\
Amiodarone & 8 \\
Hypertension & 29 \\
Coronary heart disease & 11 \\
No structural heart disease & 58 \\
Left ventricular ejection fraction $(\%)$ & $61 \pm 6(53-77)$ \\
\hline
\end{tabular}

\section{Acute Results}

In the 88 patients, $354 \mathrm{PVs}$ were detected by angiography, including 13 patients with a left common trunk. PVAC could be steered to 353/354 PVs (99\%) using a nonsteerable sheath. PV potentials were observed in 339/353 PVs (96\%) either spontaneously or as a result of pacing maneuvers and were therefore targeted for antral ablation. The other veins were not treated with ablation in this study.

The detailed results of a single ablation procedure with the PVAC are summarized in Table 2. Complete PV isolation was achieved in all but 1 left inferior PV (99\%) with a mean of $24 \pm 9$ energy applications per patient.

Most of the energy applications (82\%) were administered with the patients in sinus rhythm. AF for $>60 \mathrm{~s}$ was induced during $4 \%$ of all applications of energy in the antral regions of the PVs. A spontaneous or ablation-induced episode of $\mathrm{AF}$ terminated in $11 \%$ of all patients during ablation with the PVAC. The mean number of transthoracic cardioversions was $1.2 \pm 1.1$ per patient (range $0-4$ ).

The mean procedure time was $125 \pm 28$ minutes (range 59-211 minutes); the mean fluoroscopy time for PV isolation with the PVAC was $21 \pm 13$ minutes (range 14-46 minutes). There were no acute or late procedure-related complications.

\section{Endoscopy of the Esophagus}

All 12 patients undergoing endoscopy of the esophagus $24 \mathrm{~h}$ after the ablation procedure showed normal esophageal mucosa.

TABLE 2

Characteristics of PVs and PV Isolation

\begin{tabular}{lc}
\hline & N (\%), [range] \\
\hline Procedures & 88 \\
Total PVs & 354 \\
PVs reached with PVAC & $353 / 354(99 \%)$ \\
Targeted veins showing PV potentials & $339 / 353(96 \%)$ \\
Successful PV isolation with PVAC & $338 / 339(99 \%)$ \\
Mean no. of RF applications & $24 \pm 9[10-46]$ \\
Mean no. of external cardioversions & $1.2 \pm 1.1[0-4]$ \\
Procedure time (minute) & $125 \pm 28[59-211]$ \\
Fluoroscopy time (minute) & $21 \pm 13[14-46]$ \\
Complications & $0 / 88$ \\
\hline PV = pulmonary vein. &
\end{tabular}

TABLE 3

Follow-Up Results

\begin{tabular}{lcc}
\hline & $\begin{array}{c}\text { Group 1 } \\
\mathbf{7 3} \text { and } \\
\mathbf{n}=\mathbf{1 3} \text { Months }\end{array}$ & $\begin{array}{c}\text { Group 2 } \\
\mathbf{1 2} \text { Months } \\
\mathbf{n = 2 4}\end{array}$ \\
\hline Freedom from AF & $60(82.2 \%)$ & $19(79.2 \%)$ \\
AF/failure & 13 & 5 \\
Redo procedures & $6(8.2 \%)$ & $1(4.2 \%)$ \\
No intervention & 7 & 2 \\
\hline
\end{tabular}

\section{Follow-Up Results}

In group 1, recurrence of AF, detected by Holter monitoring and/or by 12 lead ECG because of symptomatic ongoing palpitations, was observed in 13 of 73 patients within a mean follow-up period of $4.8 \pm 1.5$ months, while freedom of AF was found in $82.2 \%$ of them. Seven patients decided to have a redo procedure, while 7 patients declined because the episodes were asymptomatic $(n=3)$, brief $(n=4)$, and/or infrequent $(\mathrm{n}=3)$.

In group 2, 3 of the 24 patients still had AF episodes at 12 months of follow-up. Two other patients in this group had already undergone a redo procedure, while 19 patients were in sinus rhythm with freedom from AF (79.2\%). A second ablation procedure was performed in 1 patient who was known to have recurrent AF by earlier follow-up evaluation (Table 3).

There were no episodes of left atrial tachycardia or left atrial flutter detected in any of the patients during followup. In all patients undergoing a second procedure, we found varying patterns of $\mathrm{PV}$ reconnection(s) to the left atrium. Angiography of the PVs showed no evidence of PV stenosis in these patients.

In the subgroup of patients who completed all follow-up visits $(\mathrm{n}=24)$, freedom from $\mathrm{AF}$ was observed in $79 \%$ at 3 -month, $83 \%$ at 6 -month, and $79 \%$ at 12 -month follow-up, respectively.

\section{MR Imaging}

None of the 18 patients with MRI at 6-month follow-up had any evidence of hemodynamically relevant PV stenosis.

\section{Discussion}

In this study, a cohort of patients with paroxysmal AF was treated with a decapolar spiral catheter system that delivers RF energy in a bipolar/unipolar fashion. It can be used for both mapping and ablation and eliminates the need for 3-D imaging tools or remote navigation.

\section{Acute Efficacy}

Current approaches utilized for PV mapping and ablation require multielectrode mapping catheters or sophisticated mapping systems and result in approximately $65-85 \%$ long-term success. ${ }^{8-11}$ Limitations of this approach include prolonged learning curves, the need for more than 1 catheter in the left atrium, prolonged procedure times, and significant risk of complications. Alternatively, a single conventional catheter in conjunction with an electroanatomical mapping system has been successfully used for circular and linear point-by-point RF delivery in patients with $\mathrm{AF}^{10,12}$ With 
our approach, $99 \%$ of all targeted PVs were isolated with one mapping/ablation catheter without the use of a steerable sheath in a series of 88 consecutive patients.

Our acute success rate compares favorably with the results presented by other investigators. In a study by Boersma et al., $100 \%$ of PVs were successfully isolated using the same system in a series of 98 patients. ${ }^{1}$ Using conventionaltip catheters, Cheema et al. reported a $75 \%$ success rate, ${ }^{13,14}$ while Hocini et al. and Verma et al. report $100 \%$ success rates with the isolation of PVs. ${ }^{15-17}$

Various investigational devices utilizing ultrasound, laser, or cryoenergy have been evaluated to simplify PV electrical isolation procedures. ${ }^{18-21}$ These devices are not designed for mapping, and an additional catheter is required to evaluate for PV isolation. A mesh catheter can overcome these limitations. This catheter, which is placed in the region of the PV antrum, is able to deliver RF energy in a pulsed, temperature-control mode over selected electrodes and map the corresponding electrical activity in the region of the PV ostium. Initial clinical experience with the mesh catheter was recently reported by Steinwender et al. ${ }^{22}$ These investigators reported a $97 \%$ success rate for $\mathrm{PV}$ isolation of all targeted veins in a group of 26 patients, with a mean fluoroscopy and procedure time of $34.6 \pm 10.0$ and $187 \pm 36$ minutes, respectively. $^{22}$

\section{Ablation Strategy and Follow-Up Results}

Our ablation strategy differs from the approach of Boersma et al., who used the same ablation system. ${ }^{1}$ Only those veins with spontaneous or stimulation-induced PV potentials were targeted for antral ablation in our series. This resulted in RF ablation of $96 \%$ of all PVs. Other investigators have limited PV isolation to potentially arrhythmogenic PVs as well. ${ }^{22}$ Despite this difference, the acute results and the clinical course during follow-up were very similar: after 6 months, $83 \%$ of all patients in the study of Boersma were free from $\mathrm{AF}^{1}{ }^{1}$ while 82 and $79 \%$ of our patients off antiarrhythmic drugs did not show AF after a mean follow-up of 5 months and 1 year, respectively.

This is the first study with the PVAC presenting 1-year clinical results in a subgroup of patients after PV isolation. We found a slight decrease in freedom from AF at 12-month follow-up, with $79 \%$ of patients in sinus rhythm. It seems that in most patients, the early results after ablation remain stable over time. Comparable results were seen when only the first 24 patients who completed all follow-up visits are analyzed. Other investigators have published similar data, reporting freedom from $\mathrm{AF}$ in 56 and $92 \%$ of patients, depending on the ablation strategy and intensity of follow-up. $8,12,13,23$

\section{Procedure Times}

The mean total procedure time from femoral vein access to complete catheter withdrawal was $125 \pm 28$ minutes, with a fluoroscopy time of $21 \pm 13$ minutes. While the fluoroscopy time differs only slightly from that of Boersma et al. (18 \pm 8 minutes), a significantly shorter mean procedure time (84 \pm 29 minutes) was reported by this group. ${ }^{1}$ The reasons for this difference might be the PV mapping maneuvers used in our study: to clearly differentiate between residual PV conduction from far-field signals in the region of the PV antrum or PV ostium, we paced in the CS, LAA, posterior LA, and different sites in the right atrium.
With conventional single-tip catheters, a wide range of procedural and fluoroscopy times have been reported for complete PV isolation: 70 (Hocini et al.) ${ }^{15}$ to 256 (Karch et al. $)^{24}$ minutes procedure time and 18-72 minutes fluoroscopy time, respectively. Many of these studies used an electroanatomical 3-D mapping system for guiding the ablation procedure. Using alternative energy sources such as cryoenergy resulted in a total procedure time of $211 \pm 108$ minutes with a mean fluoroscopy time of $52 \pm 36$ minutes. ${ }^{25}$ Significantly, shorter procedure and fluoroscopy times were reported with the mesh catheter in 26 patients for PV isolation. ${ }^{22,26}$

The PVAC isolation technique compares favorably to conventional and investigational techniques, with significantly shorter procedure and fluoroscopy times, and with no requirement for 3-D mapping systems.

\section{Safety}

There were no procedure-related complications in this study. In the studies by Boersma et al. and Fredersdorf et al., no complications occurred in series of 98 and 21 patients, using the same technology. 1,27 A worldwide survey on the methods and safety of conventional catheter ablation for AF published in 2005 reported an incidence of major complications of $6 \% .^{23}$

Endoscopy of the esophagus performed 24 hours after ablation revealed esophageal wall injuries in $47 \%$ of patients undergoing PV antrum isolation with conventional $8 \mathrm{~mm}$ and irrigated tip electrodes. ${ }^{28}$ In contrast, in the last 12 patients in this study, there were no esophageal lesions in any patient. By using alternating bipolar/unipolar RF energy with a power maximum of $10 \mathrm{~W}$, the risk of collateral damage to the surrounding structures (phrenic nerve, esophagus) ) $^{28,29}$ may be minimized.

We found no evidence for hemodynamically relevant PV stenosis with MR imaging in 18 patients at 6-months followup and/or angiographically in 7 patients with a redo procedure. Similar data were presented by Boersma et al. and Fredersdorf et al. in 19 and 21 patients, respectively. ${ }^{1,27}$

\section{Limitations}

Although an ablation efficacy of 82 and $79 \%$ after a mean follow-up of 5 and 12 months is an encouraging result, longer follow-up periods with more patients are necessary, since the effect of catheter ablation has been shown to decline over time in some patients. ${ }^{30}$ The number of patients reaching 1 year follow-up is still too small for a final statement on the clinical efficacy of the method described. No data dealing with the potential issue of PV stenosis ${ }^{31}$ are available for all patients in this study. However, in other studies using the same device for antral ablation, there was no evidence of PV stenosis at MRI follow-up or angiographically. ${ }^{1,27}$

\section{Conclusions}

PV isolation with the multiarray PVAC system in conjunction with low-power phased RF energy appears to be safe and feasible, with a promising clinical efficacy that appears stable over time. Compared to other ablation systems in clinical and investigational use, the procedure and fluoroscopy times appear to be relatively shorter. 
Acknowledgments: The authors thank Mr. Jay Kelley for reviewing the manuscript.

\section{References}

1. Boersma LV, Wijffels MC, Oral H, Wever EF, Morady F: Pulmonary vein isolation by duty-cycled bipolar and unipolar radiofrequency energy with a multielectrode ablation catheter. Heart Rhythm 2008;5:1635-1642.

2. Bugge E, Nicholson IA, Thomas SP: Comparison of bipolar and unipolar radiofrequency ablation in an in vivo experimental model. Eur $\mathrm{J}$ Cardiothorac Surg 2005;28:76-80.

3. Michaud G, Martin D, John R: Safety using novel multi-array catheters and phased radiofrequency energy in left atrial ablation for persistent atrial fibrillation (abstract). Heart Rhythm 2008;5(Suppl):S313-S314.

4. Scharf C, Boersma LVA, Kanagaratnam P: Long term efficacy when using multi-array catheters and phased radiofrequency energy for ablation of chronic atrial fibrillation (abstract). J Am Coll Cardiol 2008; 51:A8.

5. Wijffels MC, Oosterhout M, Vos M: Characterization of atrial lesions using multipolar ablation catheters in pigs (Abstract Suppl). Eur Heart J 2008;29:412.

6. Wijffels MC, VAN Oosterhout M, Boersma LV, Werneth R, Kunis $\mathrm{C}, \mathrm{Hu} \mathrm{B}, \mathrm{Beekman} \mathrm{JD}$, Vos MA: Characterization of in vitro and in vivo lesions made by a novel multichannel ablation generator and a circumlinear decapolar ablation catheter. J Cardiovasc Electrophysiol 2009;20:1142-1148.

7. Iwasa A, Storey A, Tashakkor B, Feld K: Identification of pulmonary vein potentials by differential site atrial pacing in patients with paroxysmal atrial fibrillation: Enhanced detection by pulmonary vein pacing. J Cardiovasc Electrophysiol 2003;14:1311-1318.

8. Arentz T, von Rosenthal J, Blum T, Stockinger J, Burkle G, Weber R, Jander N, Neumann FJ, Kalusche D: Feasibility and safety of pulmonary vein isolation using a new mapping and navigation system in patients with refractory atrial fibrillation. Circulation 2003;108:2484-2490.

9. Arentz T, von Rosenthal J, Weber R, Burkle G, Blum T, Stockinger J, Neumann FJ, Kalusche D: Effects of circumferential ostial radiofrequency lesions on pulmonary vein activation recorded with a multipolar basket catheter. J Cardiovasc Electrophysiol 2005;16:302-308.

10. Pappone C, Rosanio S, Oreto G, Tocchi M, Gugliotta F, Vicedomini G, Salvati A, Dicandia C, Mazzone P, Santinelli V, Gulletta S, Chierchia S: Circumferential radiofrequency ablation of pulmonary vein ostia: A new anatomic approach for curing atrial fibrillation. Circulation 2000;102:2619-2628

11. Tondo C, Mantica M, Russo G, Karapatsoudi E, Lucchina A, Nigro F, Wild M, Molinaro C, Bavila R: A new nonfluoroscopic navigation system to guide pulmonary vein isolation. Pacing Clin Electrophysiol 2005;28(Suppl 1):S102-S105.

12. Pappone C, Rosanio S, Oreto G, Tocchi M, Gugliotta F, Salvati A, Dicandia C, Mazzone P, Santinelli V, Gulletta S, Vicedomini G: Prospects of the treatment of atrial fibrillation. Circumferential radiofrequency ablation of pulmonary vein ostia. Recenti Prog Med 2001;92:508-512.

13. Cheema A, Vasamreddy CR, Dalal D, Marine JE, Dong J, Henrikson CA, Spragg D, Cheng A, Nazarian S, Sinha S, Halperin H, Berger R, Calkins H: Long-term single procedure efficacy of catheter ablation of atrial fibrillation. J Interv Card Electrophysiol 2006;15:145-155.

14. Cheema A, Dong J, Dalal D, Vasamreddy CR, Marine JE, Henrikson CA, Spragg D, Cheng A, Nazarian S, Sinha S, Halperin H, Berger R, Calkins H: Long-term safety and efficacy of circumferential ablation with pulmonary vein isolation. J Cardiovasc Electrophysiol 2006;17:1080-1085.

15. Hocini M, Sanders P, Jais P, Hsu LF, Takahashi Y, Rotter M, Clementy $\mathrm{J}$, Haissaguerre M: Techniques for curative treatment of atrial fibrillation. J Cardiovasc Electrophysiol 2004;15:1467-1471.

16. Hocini M, Sanders P, Jais P, Hsu LF, Weerasoriya R, Scavee C, Takahashi Y, Rotter M, Raybaud F, Macle L, Clementy J, Haissaguerre $\mathrm{M}$ : Prevalence of pulmonary vein disconnection after anatomical abla- tion for atrial fibrillation: Consequences of wide atrial encircling of the pulmonary veins. Eur Heart J 2005;26:696-704.

17. Verma A, Patel D, Famy T, Martin DO, Burkhardt JD, Elayi SC, Lakkireddy D, Wazni O, Cummings J, Schweikert RA, Saliba W, Tchou PJ, Natale A: Efficacy of adjuvant anterior left atrial ablation during intracardiac echocardiography-guided pulmonary vein antrum isolation for atrial fibrillation. J Cardiovasc Electrophysiol 2007;18:151156.

18. Natale A, Pisano E, Shewchik J, Bash D, Fanelli R, Potenza D, Santarelli P, Schweikert R, White R, Saliba W, Kanagaratnam L, Tchou P, Lesh M: First human experience with pulmonary vein isolation using a through-the-balloon circumferential ultrasound ablation system for recurrent atrial fibrillation. Circulation 2000;102:1879-1882.

19. Reddy VY, Houghtaling C, Fallon J, Fischer G, Farr N, Clarke J, McIntyre J, Sinofsky E, Ruskin JN, Keane D: Use of a diode laser balloon ablation catheter to generate circumferential pulmonary venous lesions in an open-thoracotomy caprine model. Pacing Clin Electrophysiol 2004:27:52-57.

20. Saliba W, Wilber D, Packer D, Marrouche N, Schweikert R, Pisano E, Shewchik J, Bash D, Fanelli R, Potenza D, Santarelli P, Tchou P, Natale A: Circumferential ultrasound ablation for pulmonary vein isolation: Analysis of acute and chronic failures. J Cardiovasc Electrophysiol 2002;13:957-961

21. Wong T, Markides V, Peters NS, Davies DW: Percutaneous pulmonary vein cryoablation to treat atrial fibrillation. J Interv Card Electrophysiol 2004:11:117-126.

22. Steinwender C, Honig S, Leisch F, Hofmann R: Acute results of pulmonary vein isolation in patients with paroxysmal atrial fibrillation using a single mesh catheter. J Cardiovasc Electrophysiol 2008;20:147152.

23. Cappato R, Calkins H, Chen SA, Davies W, Iesaka Y, Kalman J, Kim YH, Klein G, Packer D, Skanes A: Worldwide survey on the methods, efficacy, and safety of catheter ablation for human atrial fibrillation. Circulation 2005;111:1100-1105.

24. Karch MR, Zrenner B, Deisenhofer I, Schreieck J, Ndrepepa G, Dong J, Lamprecht K, Barthel P, Luciani E, Schomig A, Schmitt C: Freedom from atrial tachyarrhythmias after catheter ablation of atrial fibrillation: A randomized comparison between 2 current ablation strategies. Circulation 2005;111:2875-2880.

25. Van Belle Y, Janse P, Rivero-Ayerza MJ, Thornton AS, Jessurun ER, Theuns D, Jordaens L: Pulmonary vein isolation using an occluding cryoballoon for circumferential ablation: Feasibility, complications, and short-term outcome. Eur Heart J 2007;28:2231-2237.

26. Meissner A, Plehn G, van Bracht M, Schrage MO, Christ M, Maagh P, Butz T, Borchard R, Trappe HJ: First experiences for pulmonary vein isolation with the high-density mesh ablator (HDMA): A novel mesh electrode catheter for both mapping and radiofrequency delivery in a single unit. J Cardiovasc Electrophysiol 2009;20:359-366.

27. Fredersdorf S, Weber S, Jilek C, Heinicke N, VON Bary C, Jungbauer C, Riegger GA, Hamer OW, Jeron A: Safe and rapid isolation of pulmonary veins using a novel circular ablation catheter and duty-cycled RF generator. J Cardiovasc Electrophysiol 2009;20:1097-1101.

28. Schmidt M, Nolker G, Marschang H, Gutleben KJ, Schibgilla V, Rittger H, Sinha AM, Ritscher G, Mayer D, Brachmann J, Marrouche NF: Incidence of oesophageal wall injury post-pulmonary vein antrum isolation for treatment of patients with atrial fibrillation. Europace 2008;10:205209.

29. Sacher F, Monahan KH, Thomas SP, Davidson N, Adragao P, Sanders P, Hocini M, Takahashi Y, Rotter M, Rostock T, Hsu LF, Clementy J, Haissaguerre M, Ross DL, Packer DL, Jais P: Phrenic nerve injury after atrial fibrillation catheter ablation: Characterization and outcome in a multicenter study. J Am Coll Cardiol 2006;47:2498-2503.

30. Shah AN, Mittal S, Sichrovsky TC, Cotiga D, Arshad A, Maleki K, Pierce WJ, Steinberg JS: Long-term outcome following successful pulmonary vein isolation: Pattern and prediction of very late recurrence. $\mathrm{J}$ Cardiovasc Electrophysiol 2008;19:661-667.

31. Robbins IM, Colvin EV, Doyle TP, Kemp WE, Loyd JE, McMahon WS, Kay GN: Pulmonary vein stenosis after catheter ablation of atrial fibrillation. Circulation 1998;98:1769-1775. 